\title{
Emotismo: Um aplicativo para auxiliar crianças no espectro autista a reconhecer e reproduzir emoções
}

\author{
Gustavo M. Nunes, Yuska Paola Costa Aguiar \\ Departamento de Ciências Exatas - Universidade Federal da Paraíba (UFPB) \\ Rio Tinto - PB - Brazil \\ \{gustavo.maciel, yuska\}@dcx.ufpb.br

\begin{abstract}
Emotismo is a mobile application to assist children with Autism Spectrum Disorder in the recognition and proper use of facial expressions to represent primary emotions. It has three main activities: recognition of emotions from socio-emotional settings, imitation of emotions with facial expressions verified via digital image recognition, and identification of emotion among several presented. Emotismo provides personalization of socioemotional contexts and the recording of usage and performance metrics. Its development considered the analysis of similar solutions and recommendations for the development of digital resources for individuals with autism and/or cognitive and learning disabilities.
\end{abstract}

Resumo. Resumo. O Emotismo é um aplicativo mobile para auxiliar crianças com Transtorno do Espectro Autista no reconhecimento e uso adequado de expressões faciais para representar as emoções primárias. Ele dispõe de três atividades principais: reconhecimento das emoções a partir de cenários socioemocionais, imitação das emoções com expressões faciais verificadas via reconhecimento digital de imagem e identificação de uma emoção dentre várias apresentadas. Ele provê personalização dos contextos socioemocionais e o registro de métricas de uso e desempenho. Seu desenvolvimento considerou análise de soluções similares e recomendações para o desenvolvimento de software para indivíduos com autismo elou déficit cognitivo e de aprendizagem.

\section{Introdução}

O Manual Diagnóstico e Estatístico de Transtornos Mentais (DSM-5) [APA, 2013] indica como uma das características do Transtorno do Espectro Autista (TEA) o déficit persistente na comunicação (verbal e não-verbal) e interação social em múltiplos contextos. Como uma das consequências, tem-se o prejuízo nas interações sociais desses indivíduos, que enfrentam barreiras para desenvolver, manter e compreender relacionamentos [Dapogny et al., 2019]. Este cenário é transversal à vida do indivíduo com autismo e causa prejuízo no seu funcionamento social, profissional e afetivo. Um dos elementos que se destaca neste contexto, é a dificuldade de interpretação e reprodução adequada de expressões faciais quando associadas às emoções. Segundo Dapogny et al. (2019), reconhecer e reproduzir corretamente expressões faciais são componentes-chave para a comunicação emocional e permitem que as pessoas expressem e entendam emoções.

Uma das estratégias para trabalhar as emoções com o público com autismo consiste na apresentação de expressões faciais, dando significado a estas em um contexto emocional pertinente. Em seguida, o indivíduo com autismo é estimulado a imitar as 
emoções apresentadas. Diversos estudos investigam a relação entre o autismo e a dificuldade em reconhecer emoções: comparações na performance do reconhecimento de emoções entre crianças neurotípicas ${ }^{1}$ e crianças neurodiversas [Uljarevic e Hamilton 2013]; a proposição de uma abordagem de aprendizado de máquina para discriminar expressões faciais e avaliar a qualidade da demonstração emocional dentro do contexto do autismo [Dapogny et al., 2019]; a criação de jogos para auxiliar no aprendizado da reprodução de emoções através de expressões faciais [Dantas et al. 2019; Dapogny et al. 2018], dentre outros. O número de estudos feitos em torno dessa relação, e o fato destes serem recentes, são indicativos da atualidade do tema e consequente necessidade de ampliar pesquisas nessa área de conhecimento.

A metodologia Applied Behavior Analysis (ABA) recomenda que as intervenções para desenvolver competências diversas pelos indivíduos no espectro devem ser personalizadas, decompostas em atividades mais simples e que estas possam ser repetidas inúmeras vezes até que sejam integralizadas no seu comportamento [Foxx, 2008]. Estas características macro são de fácil implementação nos recursos digitais, que podem ser mediadores no processo de execução de atividades junto ao público com autismo [Ploog et al., 2013], pois, além de ajudar no aumento da motivação, elas geram alguns efeitos benéficos como: atratividade, interesse, divertimento, etc. Ainda, principalmente para tablets e smartphones, a mobilidade, portabilidade e a possibilidade de personalização são importantes pois, juntas, permitem configurar as mídias apresentadas na aplicação, fazendo com que o aprendizado seja moldado dentro do contexto do indivíduo, facilitando a intervenção ao permitir que está aconteça dentro e fora do ambiente terapêutico.

Logo, o uso de tecnologias como suporte para as intervenções educacionais e práticas terapêuticas para com as pessoas no espectro é uma realidade cada vez mais comum sendo recursos úteis para melhorar a qualidade de vida cotidiana desses indivíduos [Garnier 2017]. Em adicional, o binômio "Autismo e Recursos Digitais" [Virnes, Kärnä e Vellonen, 2015] e a tríade "Autismo, Recursos Digitais e Educação" [Gonçalves et al 2019; Ferreira et al. 2018] tem sido sujeito de discussão.

Diante do exposto, neste artigo descreve-se o Emotismo ${ }^{2}$, um aplicativo Android, para apoiar as práticas que auxiliem o desenvolvimento da competência de reconhecer e usar apropriadamente expressões faciais para representação das emoções por crianças com autismo. O aplicativo é composto por três atividades principais, uma para reconhecimento das emoções (treinamento), outra para imitação das mesmas (reprodução) e uma terceira para identificação de uma emoção dentre várias apresentadas (aprendizado/integração). A partir do seu uso é possível personalizar os cenários socioemocionais para direcionar as atividades e intervenções às necessidades dos usuários. Ainda, provê o registro de tempo de uso e desempenho na realização das atividades permitindo acompanhar a evolução desta competência.

\section{Fundamentação Teórica}

As emoções são ocorrências complexas e multidimensionais, que compreendem desde fenômenos biológicos até casos subjetivos e sociais [Andrade et al.2013]. As emoções possuem função social e papel decisivo no processo da interação [Santos, 2007], pois durante as interações sociais, as pessoas avaliam e interpretam constantemente, nos outros

\footnotetext{
${ }^{1}$ Termo comumente utilizado para se referir a indivíduos fora do espectro

${ }^{2}$ APK do Emotismo
} 
indivíduos e em si mesmas, as reações emocionais expressadas. Isso acontece, normalmente, de maneira subconsciente, o que faz com que as emoções e suas formas de expressão tenham papel crucial na manutenção das relações sociais [Andrade et al. 2013].

As emoções primárias (ou básicas) [Ekman, 1999] - alegria, tristeza, medo, raiva, surpresa e desgosto - são expressadas de forma universal. Sua manifestação, via expressões faciais, segundo Ekman e Friesen (2003) e Du, Tao e Martinez (2014), podem ser descritas como segue: (i) alegria: sorriso, gerando o levantar das bochechas, além da separação dos lábios superior e inferior com consequente aparecimento das gengivas e enrugamento das pálpebras; (ii) raiva: junção das sobrancelhas ao centro, com enrugamento entre elas, olhar concentrado, na parte inferior ocorre a compressão dos lábios deixando-os mais finos; (iii) desgosto (semelhante à raiva), junção das sobrancelhas, torção do nariz e pressionamento dos lábios; (iv) tristeza: levantamento dos cantos internos das sobrancelhas unindo-as, rebaixamento do olhar e pálpebras superiores se curvam ou pendem; leve abertura da boca cujos cantos projetam-se para baixo, as bochechas são erguidas aparentando apertar os olhos; (v) medo e surpresa possuem expressões faciais semelhantes: sobrancelhas erguidas e pálpebras superiores e inferiores tensionadas, além de maxilar aberto. A principal diferença encontra-se no fato de que no medo a boca fica esticada horizontalmente e na surpresa o maxilar fica bem aberto.

Pessoas com autismo, principalmente as crianças, enfrentam barreiras em seu desenvolvimento emocional, em consequência da dificuldade para compreender os contextos sociais das emoções [Fan et al. 2018]. De acordo com Golan et al. (2010), os processos atencionais, perceptivos, cognitivos e neurais são diretamente relacionados com o contexto da compreensão das emoções, e, indivíduos no espectro processam rostos de forma diferente e mostram atenção reduzida a rostos e expressões faciais. Logo, podendo haver prejuízo nas habilidades ao processar, reconhecer e reproduzir adequadamente expressões faciais. Assim, a percepção e reconhecimento deficientes de emoções podem levar à comunicação contextualmente inapropriada ou insuficiente da emoção, podendo gerar um atraso nas competências sociais [Izard 2001]. Portanto, a dificuldade enfrentada por indivíduos autistas está ligada à importância da projeção e do reconhecimento de emoções na comunicação interpessoal [Begeer et al., 2008].

\section{Trabalhos Relacionados}

A literatura dispõe de estudos que descrevem aplicativos, jogos ou sistemas cujo objetivo de uso é direcionado ao reconhecimento de emoções por pessoas com autismo, como por exemplo: EmoStory [Fan et al, 2018], JEMImE [Dapogny et al, 2018], Michelzinho [Dantas et al. 2019], e FazeMaze [Gordon, 2014]. No entanto, destes, apenas o Michelzinho dispõe de uma versão disponível para download e uso. Tendo em vistas ampliar a análise de soluções similares ao Emotismo, foi realizada uma busca exploratória no repositório Google Play com os termos: "Autism AND Emotion" e "Autism AND Emotion AND Children". Além do Michelzinho, dois outros aplicativos foram encontrados e se tornaram alvo da análise descrita a seguir, são eles: Autimo e Emotion Learning. Vale destacar que as três soluções analisadas são jogos para trabalhar as competências emocionais e sociais em pessoas com autismo, disponíveis para plataforma móvel (tablet e smartphone). 
$\underline{\text { Michelzinho }}^{3}$ [Dantas et al. 2019] dispõe do módulo de treinamento composto pelas atividades de reconhecer e expressar emoções. No módulo jogar, a atividade expressar se repete para diferentes emoções. $\mathrm{O}$ aplicativo dispõe de dicas em áudio (as vezes de vídeo) sobre como realizar as atividades. A aplicação possui um visual inconsistente em seus diferentes módulos e não apresenta um design responsivo aos diferentes tamanhos de tela, desconsiderando as recomendações internacionais [Seeman e Cooper, 2016]. Em adicional, algumas dicas de áudio tocam simultaneamente, tornando difícil o entendimento do conteúdo. As tecnologias de Inteligência Artificial (IA) baseada em Árvore de Decisão (AD) e Rede Neural Convolucional (RNC) são adotadas para classificar, em tempo real, qual emoção o rosto do jogador está expressando. No entanto, a acurácia no reconhecimento é de $88 \%$, havendo alto índice de falha para raiva e desgosto, assim como medo e surpresa. Michelzinho é gratuito, em português e para Android.

O $\underline{\text { Autimo }}^{4}$ agrupa jogos de identificação de pares, de intrusos e jogos de adivinhação. As instruções são multimídia (texto, áudio e vídeos) e as emoções são representadas por fotos de rostos de pessoas reais. A aplicação apresenta um conjunto de características definidas como adequadas para pessoas com déficits cognitivos e de aprendizagem (como no caso do autismo), segundo as recomendações internacionais de acessibilidade [Seeman e Cooper, 2016] como: (i) design agradável, minimalista e consistente, sem elementos que possam retirar o foco do usuário; (ii) personalização de conteúdo multimídia (imagens e áudios); (iii) visualização de feedback do progresso do usuário através de gráficos e tabelas, e registro do tempo de uso para as atividades realizadas. Autimo possui uma versão gratuita (limitada), é em francês e em inglês e para iOS e Android.

Emotion Learning ${ }^{5}$ oferece uma estória com um personagem para contextualizar um cenário socioemocional e o jogador deve encontrar uma ou todas as ocorrências de uma expressão facial em meio à várias opções apresentadas. As instruções são em textos e áudio e as emoções são representadas por imagens. Sua interface possui muitas distrações visuais (background denso) e anúncios, inadequado para o público com déficit cognitivo ou de aprendizagem [Seeman e Cooper, 2016]. As representações visuais são de baixa qualidade gráfica, várias instruções incorretas são apresentadas e o conteúdo da aplicação não é personalizável. Emotion Learning é gratuito em inglês e para Android.

Diante do exposto, posicionando as características dos aplicativos descritos com o Emotismo (Quadro 1), tem-se que: como Michelzinho, se utiliza de IA para ajudar no ensino de como expressar as emoções, disponibilizado em português e de forma gratuita (assim como para o Emotion Learning); como Autimo prezamos pela consistência na aparência do aplicativo, provendo personalização de mídias, para favorecer o uso de conteúdos com os quais a criança tem familiaridade ou interesse e fornecer a visualização do progresso do usuário ao longo do uso da aplicação.

\footnotetext{
${ }^{3}$ Michelzinho, desde de abril de 2018, por Push! Sistemas, acesso em 27/11/2019

${ }^{4}$ Autimo, desde janeiro de 2015, por Auticel, acesso em 27/11/2019

${ }^{5}$ Emotion Learning, desde março de 2017, por Games ICTD, acesso em 27/11/2019
} 
IX Congresso Brasileiro de Informática na Educação (CBIE 2020)

Anais do XXXI Simpósio Brasileiro de Informática na Educação (SBIE 2020)

\begin{tabular}{|c|c|c|c|c|}
\hline \multicolumn{5}{|c|}{ Quadro 1. Comparativo entre as aplicações } \\
\hline & Autimo & Emotion Learning & Michelzinho & Emotismo \\
\hline Sistema Operacional & $\mathrm{iOS} /$ Android & Android & Android & Android \\
\hline Idioma & Inglês, Francês & Inglês & Português & Português \\
\hline Acesso & Pago & Gratuito & Gratuito & Gratuito \\
\hline Tipo de emoção & Primárias & $\begin{array}{c}\text { Primárias, exceto } \\
\text { desgosto. }\end{array}$ & Primárias & Primárias \\
\hline $\begin{array}{l}\text { Representação } \\
\text { das emoções }\end{array}$ & Fotos de pessoas & Imagens & $\begin{array}{l}\text { Fotos de } \\
\text { pessoas e } \\
\text { desenhos }\end{array}$ & $\begin{array}{c}\text { Fotos de pessoas e } \\
\text { desenhos }\end{array}$ \\
\hline Tecnologia de apoio & Não se aplica & Não se aplica & IA & IA \\
\hline Mídias & $\begin{array}{l}\text { Imagens, textos, } \\
\text { animações e áudio }\end{array}$ & $\begin{array}{c}\text { Imagens, textos e } \\
\text { áudio }\end{array}$ & $\begin{array}{l}\text { Imagens, textos, } \\
\text { animações e } \\
\text { áudio }\end{array}$ & $\begin{array}{c}\text { Imagens, textos e } \\
\text { áudio }\end{array}$ \\
\hline Personalização & $\begin{array}{l}\text { Imagens, } \\
\text { animações e } \\
\text { áudios }\end{array}$ & Não se aplica & Não se aplica & Imagens e Textos \\
\hline Registro de dados & $\begin{array}{c}\text { Progresso e tempo } \\
\text { de uso }\end{array}$ & Não se aplica & Não se aplica & $\begin{array}{l}\text { Registro de } \\
\text { desempenho }\end{array}$ \\
\hline
\end{tabular}

\section{Metodologia}

A pesquisa descrita neste artigo teve por objetivo o planejamento, o desenvolvimento e a disponibilização do jogo Emotismo para plataforma Android. Este, destina-se a crianças no espectro autista e fornece meios para reconhecimento das emoções a partir de cenários socioemocionais, imitação das emoções com expressões faciais verificadas via reconhecimento digital de imagem com IA e identificação de uma emoção dentre várias apresentadas reconhecer. Para sua execução, a metodologia aplicada consistiu no conjunto das seguintes etapas: (i) levantamento bibliográfico acerca das características e particularidades das crianças com autismo, principalmente no tocante às competências emocionais (Seção 2); (ii) análise de aplicativos com propósitos similares, para identificação de aspectos que ainda não são contemplados e que podem agregar valor à nova proposição, e para além disso, posicionar a solução proposta dentre as soluções existentes (Seção 3); (iii) planejamento e desenvolvimento do Emotismo contemplando os requisitos funcionais delimitadores do escopo da solução, passando pela definição das tecnologias para implementação do Emotismo (subseção 4.1) e incluindo levantamento e seleção de critérios e recomendações para o desenvolvimento de aplicações cujos usuários são crianças com autismo (subseção 4.2). A apresentação do Emotismo, será detalhada na Seção 5. 


\subsection{Tecnologias utilizadas no Emotismo}

Emotismo faz uso da API de processamento e análise de imagens da Luxand Cloud ${ }^{6}$, que disponibiliza um endpoint capaz de receber imagens e analisar todos os rostos encontrados, retornando as emoções expressadas. Para autenticação de usuários, armazenamento de arquivos em nuvem e banco de dados em nuvem, adotou-se os serviços do Firebase. O armazenamento em nuvens torna a personalização das imagens utilizadas no aplicativo mais fácil, assim como permite que os dados de uso (tempo e desempenho) sejam persistidos em qualquer dispositivo que acesse sua conta do aplicativo. Ainda, foi utilizado o framework Flutter, que disponibiliza um toolset para ser utilizado no desenvolvimento de aplicativos para ambos os sistemas Android e iOS.

\subsection{Recomendação para o desenvolvimento de software para usuário com autismo}

De forma a direcionar o desenvolvimento do Emotismo para que este esteja adequado para considerar a diversidade cognitiva, sensoriais e motoras das pessoas com autismo, adotou-se um conjunto de recomendações propostas pelo Cognitive and Learning Disabilities Accessibility Task Force (Coga TF) [Seeman e Cooper, 2016], a saber: apresentar o conteúdo de forma multimídia e personalizável aos interesses (motivações) particulares do usuário, apresentar uma interface minimalista, simples, consistente e livre de distrações, apresentar uma linguagem (textual e visual) adequada e de fácil compreensão, evitar que as atividades sejam finalizadas por tempo, sendo o progresso e transição entre as páginas gradual. Ainda, possuir uma configuração base pré-definida para favorecer uma personalização rápida, fácil e com pouco esforço.

\section{Emotismo}

O Emotismo é um aplicativo gratuito, mobile para plataforma Android a ser utilizado para auxiliar crianças com Transtorno do Espectro Autista no reconhecimento e uso adequado de expressões faciais para representar as emoções primárias, a falta de equipamento apropriado para desenvolvimento e testes com a plataforma iOS limitou a disponibilização do Emotismo apenas para Android. Ele dispõe de três atividades principais: reconhecimento das emoções a partir de cenários socioemocionais, imitação das emoções com expressões faciais verificadas via reconhecimento digital de imagem e identificação de uma emoção dentre várias apresentadas. O Emotismo provê personalização dos contextos socioemocionais e o registro de métricas de uso e desempenho.

Dois perfis de usuários são previstos para o Emotismo. Crianças com autismo moderado ou leve que consigam ler (usuário principal). Este tem acesso livre as funcionalidades de treinamento, imitação e identificação das emoções. Como usuário secundário previu-se a participação dos responsáveis do usuário principal (pais, cuidadores, educadores, terapeutas, e etc.) para personalizar os exercícios dispostos na atividade de treinamento das emoções, assim como para acompanhar os registros dos dados e desempenho.

Para o primeiro uso do Emotismo se faz necessário realizar um cadastro possibilitando o uso contínuo do aplicativo pelo usuário, independentemente do dispositivo em que está instalado. A tela inicial do Emotismo dispõe das três

\footnotetext{
${ }^{6}$ https://luxand.cloud/, acesso em 02/02/2020.
} 
possibilidades de atuação: (i) Jogar que permite acesso às três atividades principais da aplicação (treinamento, imitação e identificação); (ii) Ajustar as configurações que permite personalizar os itens associados a cada emoção (para a atividade de treinamento), sendo possível incluir imagens, descritivo do cenário e dicas de representação da emoção considerando um contexto familiar ou a ser trabalhado com o usuário, assim como a possibilidade de visualizar o progresso do usuário no aplicativo; e, (iii) Saber mais sobre a aplicação, onde o personagem Davi explica o funcionamento do Emotismo. O ícone de alto falante ao longo da aplicação indica a possibilidade de reproduzir em áudio os conteúdos textuais, guiando os usuários e promovendo sua autonomia no uso.

Ao clicar no botão "clique para jogar" o usuário é direcionado para as opções: “clique para treinar" e "clique para avaliar". A primeira opção irá levar o usuário para o workflow padrão da aplicação, onde o usuário deverá escolher uma emoção (Figura 1). Ao escolher uma emoção o usuário irá para as telas de reconhecimento da emoção (treinamento), imitação da emoção (reprodução) e identificação de uma emoção dentre várias apresentadas (aprendizado/integração), todas atreladas a emoção selecionada. A segunda opção irá levar o usuário diretamente para a tela de identificação, entretanto desta forma a função de identificação não estará ligada a somente uma emoção.

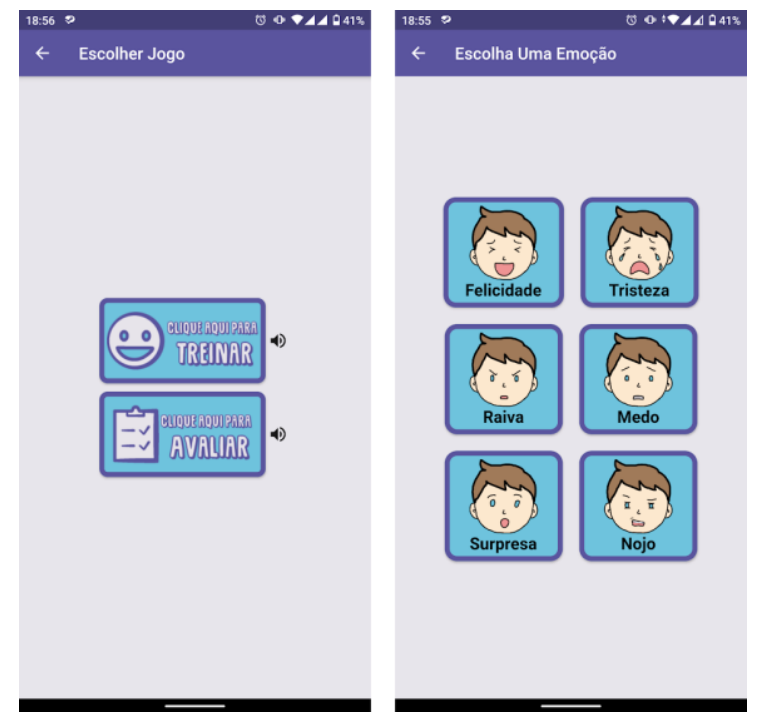

Figura 1. Screenshots das telas Escolher Jogo e Escolher Emoção

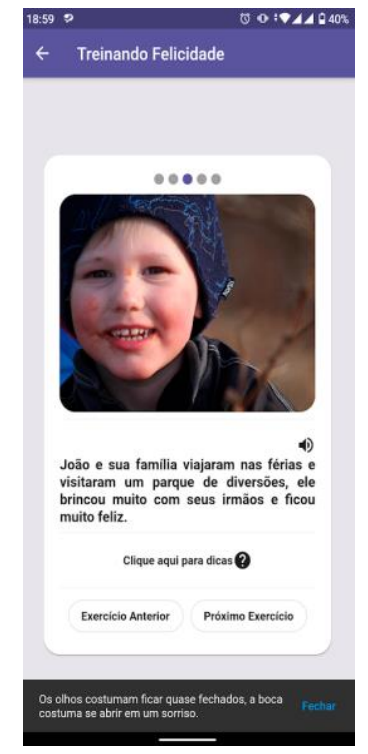

Figura 2. Treinamento das emoções

Para o reconhecimento das emoções são disponíveis cards descritivos (Figura 2) de uma situação que se encaixa como causa da emoção-alvo a ser trabalhada, incluindo uma foto de um rosto expressando tal emoção e dicas de como realizar a sua identificação a partir das expressões faciais.

$\mathrm{Na}$ atividade de imitar a emoção que acabou de ser treinada, um card é apresentado e o usuário é convidado a reproduzir, por expressão facial, a emoção que acabou de ser trabalhada. O reconhecimento das expressões faciais é seguido do feedback imediato sobre a imitação (reprodução) feita pela criança. Se adequada para a emoção alvo, o usuário é levado à atividade de identificação das emoções; se inadequado, o jogador retorna à atividade de treinamento do reconhecimento das emoções. 


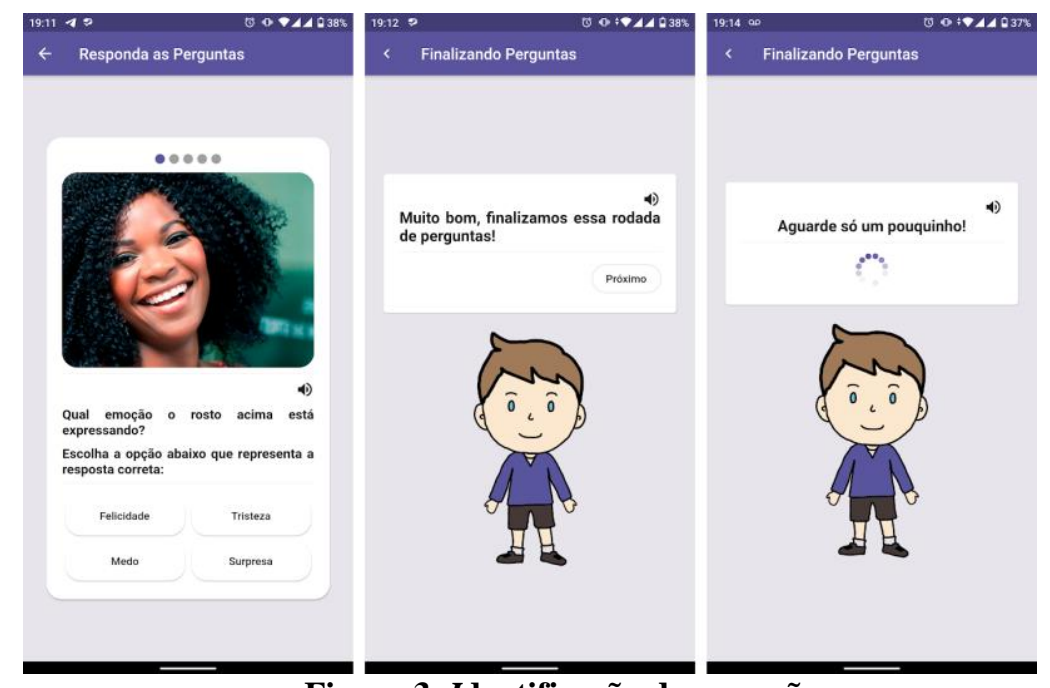

Figura 3. Identificação das emoções

A atividade de identificação de emoções é composta por um conjunto de 5 cards (apresentadas sequencialmente) onde cada um possui a foto de uma pessoa expressando a emoção alvo. O jogador deve escolher a emoção que está sendo representada (Figura 3). No acerto, o próximo card é apresentado; para o erro, um áudio será reproduzido convidado o jogador a tentar novamente. Ao chegar ao último card, o personagem Davi explicará ao usuário que os dados obtidos na atividade estão sendo salvos.

Embora o Emotismo disponha de cenários socioemocionais predefinidos (5 para cada uma das emoções primárias), é possível personalizar cenários de forma que os as estórias, imagens e dicas sejam conectadas à realidade da criança. Esta funcionalidade é destinada ao usuário secundário e adotou-se uma estratégia de bloqueio ao seu acesso (resolver uma operação matemática). Após desbloqueio, deve-se escolher a emoção a ser trabalhada e seguir com a seleção do cenário socioemocional para edição do exercício (imagem apresentada, a estória que contextualiza a emoção e as dicas do exercício).

Diante da importância de acompanhar do uso do aplicativo e do desempenho do usuário principal, as informações de uso são registradas no Emotismo e podem ser consultadas pelo usuário secundário. Este recurso é relevante para apoiar a personalização de cenários socioemocionais ao conhecer quais emoções o usuário tem apresentado maior dificuldade em identificar e/ou imitar. São registradas data e hora de realização das atividades, quantidade de tentativas até o acerto, qual era a emoção correta, e se a questão foi respondida ou não.

\section{Considerações Finais e Trabalhos Futuros}

O Emotismo é um aplicativo gratuito, em português e mobile para Android, que fornece um conjunto de atividades (personalizáveis) para que crianças com autismo possam aprimorar suas habilidades socioemocionais. Este projeto de pesquisa e desenvolvimento tem como objetivo fornecer um recurso digital para auxiliar as intervenções sobre as competências socioemocionais ligadas ao reconhecimento e imitação das emoções através do uso de expressões faciais. Não há a pretensão de substituir os métodos convencionais de intervenção, apenas servir como ferramenta de apoio aos mesmos.

A utilização de recursos tecnológicos aliado às formas de intervenção clássicas pode favorecer os resultados pretendidos junto ao público no espectro autista [Ploog et 
al, 2013]. Ainda, o uso de recursos digitais foi identificado como uma prática de intervenção emergente pelo National Standards Project [Steinbrenner et al., 2020]. Este contexto evidencia a importância de direcionar esforços para desenvolver aplicativos e softwares destinados ao público com autismo. Portanto, espera-se que a disponibilização do Emotismo possa servir como uma ferramenta importante, a ser utilizada em conjunto com outras formas de intervenção, na educação socioemocional da criança com autismo.

Durante o desenvolvimento do Emotismo, houve a preocupação em adaptar as decisões de projeto de software e de interface para acomodar as particularidades do público-alvo a partir da adoção do conjunto de recomendações internacionais do Coga TF [Seeman e Cooper, 2016]. No entanto, embora esta iniciativa aproxime a adequação da solução proposta às particularidades das crianças com autismo, tem-se consciência das limitações atuais deste projeto, uma vez que sua avaliação não foi realizada junto aos usuários potenciais: primários (pessoas com autismo) e/ou secundários (cuidadores, pais, educadores, terapeutas, etc.).

Cientes da importância de refinamento da aplicação, enquanto trabalho futuro para continuação deste projeto uma validação ampla e abrangente do Emotismo é prevista como ponto de partida. Para tanto, se faz necessário encaminhar e ter aprovação do comitê de ética, uma vez que o processo de avaliação deve contemplar a participação de especialistas e profissionais que atuam com autismo, de preferência no contexto de intervenções relacionadas às competências socioemocionais, assim como a participação de crianças com autismo.

\section{Agradecimentos}

Ao CNPq, pelo financiamento via Universal (MCTIC/CNPq No28/2018,425551/2018-0).

\section{Referências}

American Psychiatric Association (APA). (2013). Diagnostic and statistical manual of mental disorders (DSM-5). American Psychiatric Pub.

Andrade, N. C., Abreu, N. S., Duran, V. R., Veloso, T. J., \& Moreira, N. A. (2013). Reconhecimento de expressões faciais de emoções: padronização de imagens do teste de conhecimento emocional. Psico, 44(3), 382-390.

Begeer, S., Koot, H. M., Rieffe, C., Terwogt, M. M., \& Stegge, H. (2008). Emotional competence in children with autism: Diagnostic criteria and empirical evidence. Developmental Review, 28(3), 342-369.

Dantas, A. C., de Melo, S., Neves, L., Milessi, T., \& do Nascimento, M. Z. (2019). Michelzinho: Jogo sério para o ensino de habilidades emocionais em pessoas com autismo ou deficiência intelectual. Simpósio Brasileiro de Informática na Educação.

Dapogny, A et al. (2018). JEMImE: a serious game to teach children with ASD how to adequately produce facial expressions. In 2018 13th IEEE International Conference on Automatic Face \& Gesture Recognition (FG 2018) (pp. 723-730). IEEE.

Dapogny, A et al. (2019). On automatically assessing children's facial expressions quality: a study, database, and protocol. Frontiers in Computer Science, 1, 5.

Du, S., Tao, Y., \& Martinez, A. M. (2014). Compound facial expressions of emotion. Proceedings of the National Academy of Sciences, 111(15), E1454-E1462. 
Ekman, P. (1999). Basic emotions. Handbook of cognition and emotion, 98(45-60), 16.

Ekman, P., \& Friesen, W. V. (2003). Unmasking the face: A guide to recognizing emotions from facial clues. Ishk.

Fan, M., Fan, J., Jin, S., Antle, A. N., \& Pasquier, P. (2018). EmoStory: A Game-based System Supporting Children's Emotional Development. In Extended Abstracts of the 2018 CHI Conference on Human Factors in Computing Systems (pp. 1-6).

Ferreira, W., Cordeiro, R., Aguiar, Y. P. C., Saraiva, J., Tardif, C., \& Galy, E. (2018, October). Panorama das Publicações Nacionais sobre Autismo, Educação e Tecnologia. In Brazilian Symposium on Computers in Education (Simpósio Brasileiro de Informática na Educação-SBIE) (Vol. 29, No. 1, p. 913).

Foxx, R. M. (2008). Applied behavior analysis treatment of autism: The state of the art. Child and adolescent psychiatric clinics of North America, 17(4), 821-834.

Garnier, P. (2017). Autisme et Nouvelles Technologies. Revue de littérature sur les questions de l'usage des outils numériques dans l'aide à la communication non verbale et à l'interaction sociale chez les personnes avec des troubles du spectre de l'autisme (TSA).

Golan, O. et al. (2010). Enhancing emotion recognition in children with autism spectrum conditions: An intervention using animated vehicles with real emotional faces. Journal of autism and developmental disorders, 40(3), 269-279.

Gonçalves, R., Pessoa, C., Passos, O. M., \& de Amorim, R. (2019). Ferramentas Assistivas no Ensino e Aprendizagem de Crianças com Aspectro Autista: Um Mapeamento Sistemático. Simpósio Brasileiro de Informática na Educação.

Gordon, I. (2014). FaceMaze: An Embodied Cognition Approach To Facial Expression Production in Autism Spectrum Disorder (Doctoral dissertation).

Izard, C. E. (2001). Emotional intelligence or adaptive emotions?.

Ploog, B. O., Scharf, A., Nelson, D., \& Brooks, P. J. (2013). Use of computer-assisted technologies (CAT) to enhance social, communicative, and language development in children with autism spectrum disorders. Journal of autism and developmental disorders, 43(2), 301-322.

Santos, F. M. T. D. (2007). As emoções nas interações e a aprendizagem significativa. Ensaio Pesquisa em Educação em Ciências (Belo Horizonte), 9(2), 173-187.

Seeman, L., \& Cooper, M. (2016). Techniques for the the cognitive and learning disabilities accessibility task force (COGA). W3C.

Steinbrenner, J. R. et al. (2020). Evidence-based practices for children, youth, and young adults with Autism. The University of North Carolina at Chapel Hill, Frank Porter Graham Child Development Institute, National Clearinghouse on Autism Evidence and Practice Review Team.

Uljarevic, M., \& Hamilton, A. (2013). Recognition of emotions in autism: a formal metaanalysis. Journal of autism and developmental disorders, 43(7), 1517-1526.

Virnes, M., Kärnä, E., \& Vellonen, V. (2015). Review of research on children with autism spectrum disorder and the use of technology. Journal of Special Education Technology, 30(1), 13-27. 